\title{
Fault Identification Based on Wide-Azimuth Seismic Data: A Case Study in the Bohai Bay
}

\author{
Sian Zhu, Hongtao Chen, Yongjun Hu, Yubin Feng, Chunzhi Zhang \\ Dagang Branch, BGP, CNPC, Tianjin, China \\ Email: zhusian@cnpc.com.cn
}

How to cite this paper: Zhu, S., Chen, $\mathrm{H}$. T., Hu, Y. J., Feng, Y. B., \& Zhang, C. Z. (2019). Fault Identification Based on Wide-Azimuth Seismic Data: A Case Study in the Bohai Bay. Journal of Geoscience and Environment Protection, 7, 294-299. https://doi.org/10.4236/gep.2019.75021

Received: March 6, 2019 Accepted: May 28, 2019 Published: May 31, 2019

\begin{abstract}
Full-azimuth common reflection-angle gathers could be stacked at any azimuth. The stacked data at the azimuth perpendicular to fractures exhibit the largest azimuthal anisotropy, while the stacked data at the azimuth parallel to fractures exhibit the smallest azimuthal anisotropy. In petroleum exploration in Block A, a neritic province in the Bohai Bay, we use the stacked data at the azimuth perpendicular to fractures to improve fault interpretation. We also use attribute fusion to identify fractures in different directions.
\end{abstract}

\section{Keywords}

Wide Azimuth, Fault Identification, Azimuth Stack, Bohai Bay Basin, Azimuth Fusion

\section{Introduction}

A seismic survey with the aspect ratio above 0.5 is known as a wide-azimuth survey, while a survey with the aspect ration below 0.5 is called a narrow-azimuth survey (Figure 1) (Wang et al., 2015; Cordsen \& Galbraith, 2002; Baldock et al., 2012). In spite of its wide application, a narrow-azimuth seismic survey cannot render sufficient precision; instead, wide-azimuth seismic prospecting and even full-azimuth prospecting have been utilized in recent years due to increasingly proved effects. Compared with narrow-azimuth prospecting, wide-azimuth prospecting has following advantages (Hu et al., 2004; Baldock et al., 2012; Schoenberg et al., 1999). 1) There are more crossline folds and enhanced illumination to mitigate the influence of surface obstacles and subsurface shadows and improve seismic images. 2) Attribute variation with offset and azimuth (AVOA) and velocity variation with azimuth (VVA) in 3D wide-azimuth data may be used to identify azimuthal anisotropy. 3) Wide-azimuth acquisition has better spatial continuity and less noises; thus, seismic imaging and lateral 


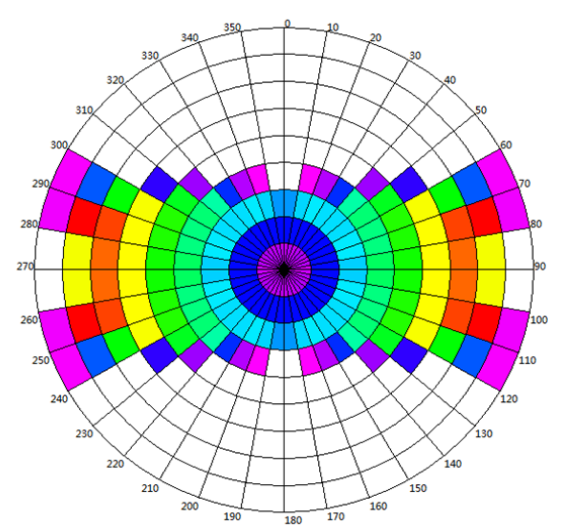

(a)

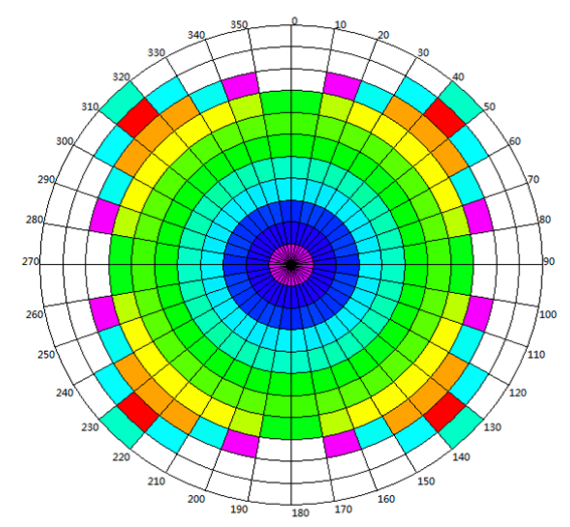

(b)

Figure 1. Azimuth-offset distribution in an acquisition system. (a) Narrow azimuth; (b) Wide azimuth.

continuity may be improved, especially for steeply dipping formations. In addition, wide-azimuth data have better amplitude preservation and resolution.

\section{Geologic Setting}

The Bohai Bay Basin lies in a coastal region in eastern China and is surrounded by the Jiaoliao Uplift, Yanshan Uplift, Taihangshan Uplift, and LuxiUplift (Lai, 2001; Zhu et al., 2001). It is a Mesozoic-Cenozoic rift basin superposed on Paleozoic cap formation in the North China Platform. The Bohai Bay Basin is an important petroliferous basin covering terrestrial and marine space in eastern China. The area of interest lies in the south of the sea area in the Bohai Sea, where there are many fractures of different grades. Major faults control fault-block structures and the size of oil and gas structures, while low-grade faults are important to local traps. This means it is important to identify fractures of different grades.

\section{Methodology and Results}

Fractures play an important role in hydrocarbon generation, migration, accumulation, and preservation. Due to the effect of multi-phase tectonic activities, there are usually several groups of fracture systems with different strikes. It is hard to interpret such fractures by using narrow-azimuth seismic data. On the contrary, we may use wide-azimuth data to generate azimuth stacks for the interpretation of structures in different directions.

\subsection{Azimuth Selection}

It is necessary to understand regional tectonic stress and fault strike in the area of interest before fault identification based on azimuth stacks. Azimuthal anisotropy has the smallest intensity in the direction parallel to fractures and the largest intensity in the direction perpendicular to fractures. Azimuthal stacks should be generated in accordance with fault strike. Each fault strike should correspond 
to an azimuthal stack.

The fractures in the area of interest mainly extend in a NE direction. Some extend in a nearly east-west direction. Thus, the azimuth stack at the NE direction was the first selection for fault interpretation. Fault points were then grouped on the map. Using several azimuth stacks, we may improve fault identification.

Figure 2 shows two azimuth stacks. As indicated by the blue arrow in Figure 2(a), the azimuth stack generated in the direction parallel to the NE major faults shows event flexure instead of discontinuity. As shown in Figure 2(b), the azimuth stack generated in the direction perpendicular to major faults exhibits distinct discontinuity of events.

Figure 3 compares two coherence slices at different azimuths. The slice in Figure 3(b) at the azimuth perpendicular to major faults has better image of a fault indicated by the blue arrow than the slice in Figure 3(a) parallel to major faults.

\subsection{Azimuth Fusion}

Fault detectability varies with azimuth. Thus, we may integrate seismic attributes at different azimuths to improve fault interpretation (James, 2006; Manning, 2007, Ling, 2003). Figure 4 shows several single-azimuth time slices and an integrated slice of different azimuths. The spatial relation of fractures indicated by the red arrow is ambiguous at azimuths 1,2 , and 4 . As shown in the slice at azimuth 3 which is perpendicular to the fault strike, this fault system consists of two separate en-echelon faults; their spatial relation agrees with regional tectonic stress which led to extensional strike-slip faults in this area. The low-grade fault extending in a NE direction indicated by the blue arrow is not clearly imaged at azimuth 1, 3, and 4, but the slice at azimuth 2 shows a clear image. Above information was integrated in Figure 4(e); hence, it is easy to identify low-grade faults in different directions, such as those indicated by the red and blue arrows. Azimuthal attribute fusion improved fault interpretation.

\subsection{Result}

Through joint interpretation of wide-azimuth seismic data, the fracture system in Block A was delineated with more credibility. Some low-grade faults, along

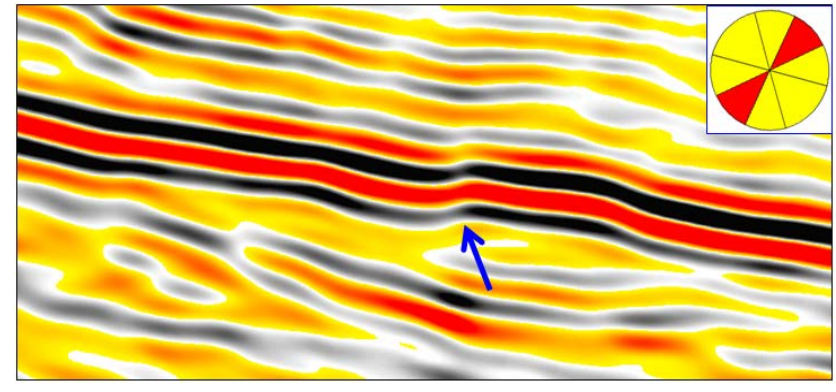

(a)

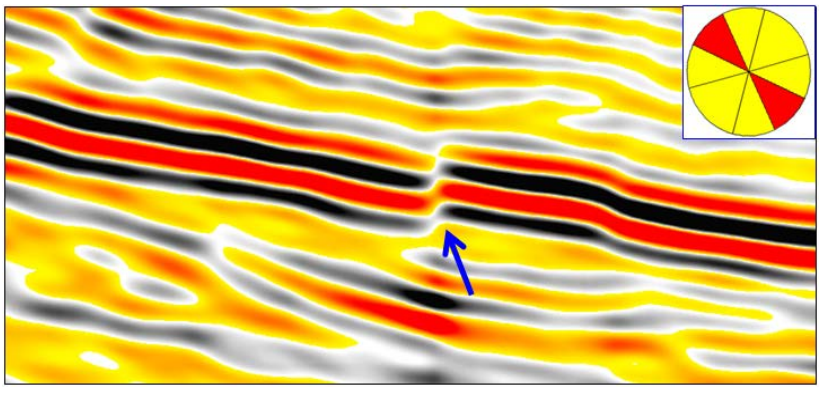

(b)

Figure 2. Azimuth stacks in Block A. (a) Azimuth 1; (b) Azimuth 2. 


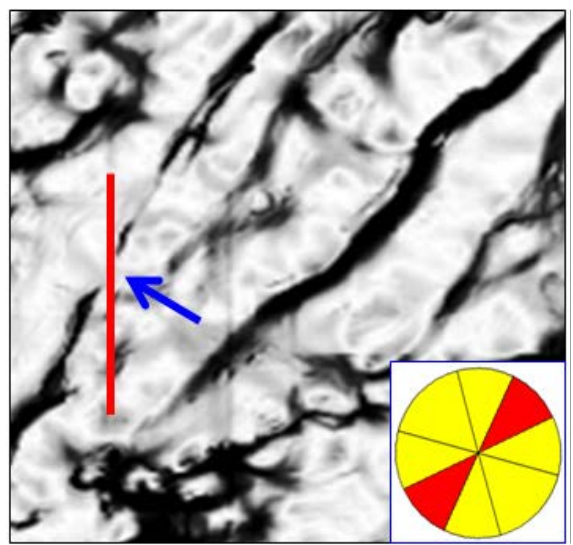

(a)

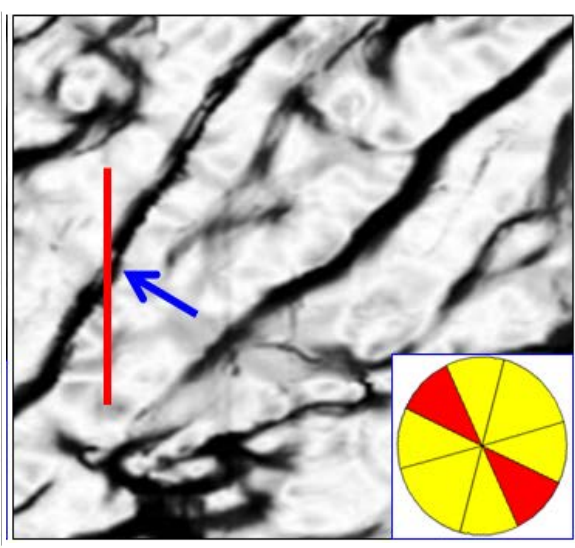

(b)

Figure 3. Coherence slices at two azimuths in Block A. (a)Azimuth 1; (b) Azimuth 2.

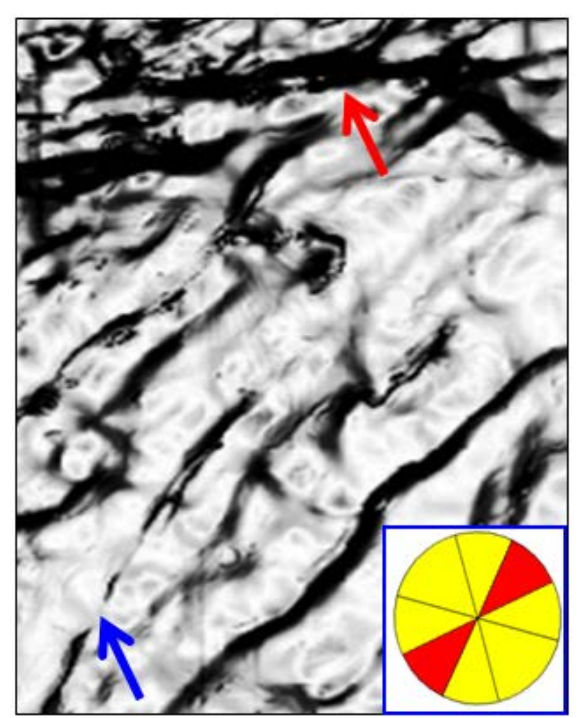

(a)

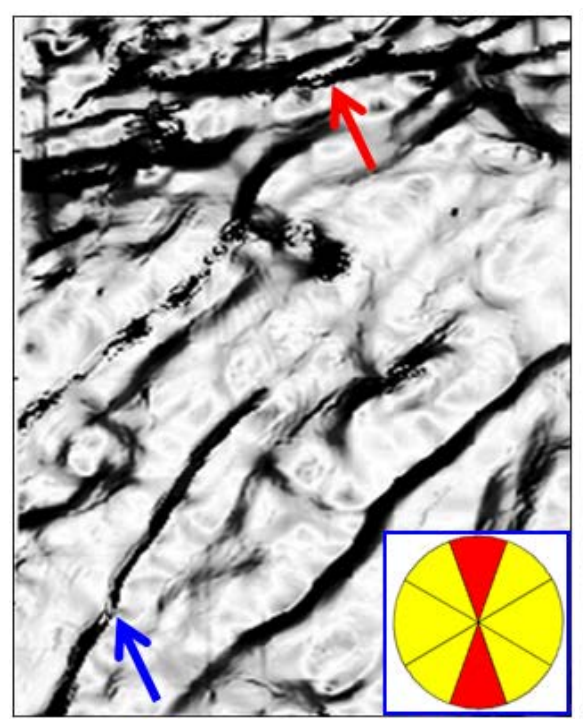

(c)

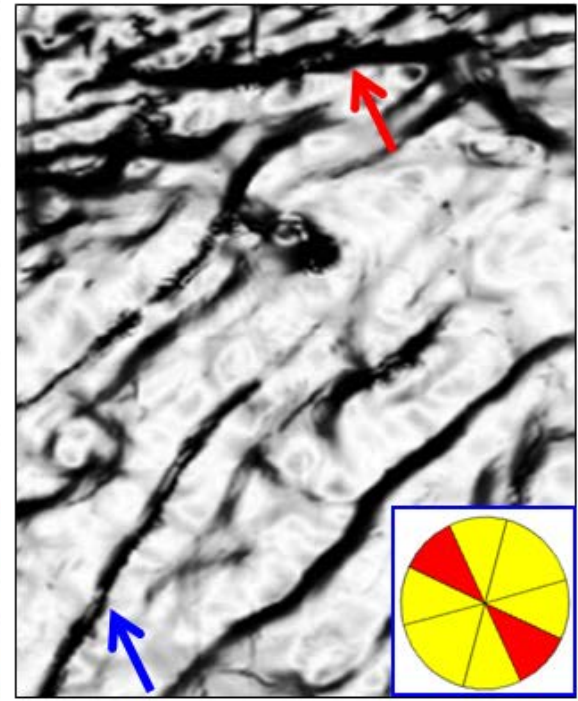

(b)

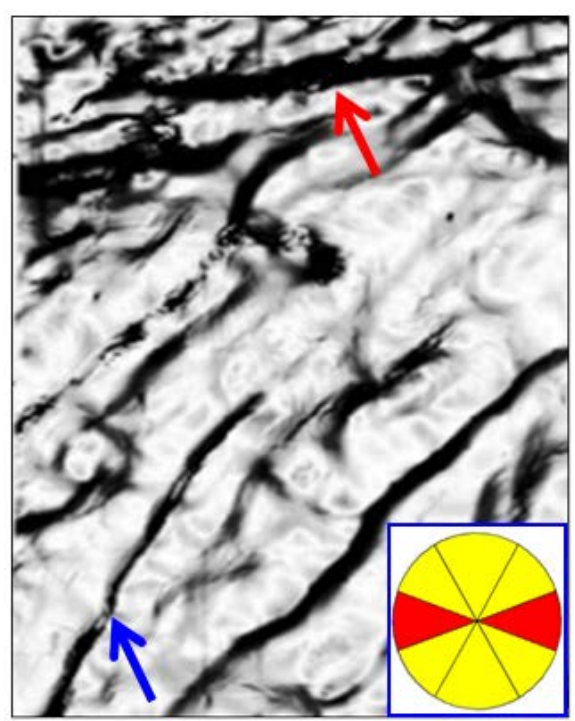

(d) 


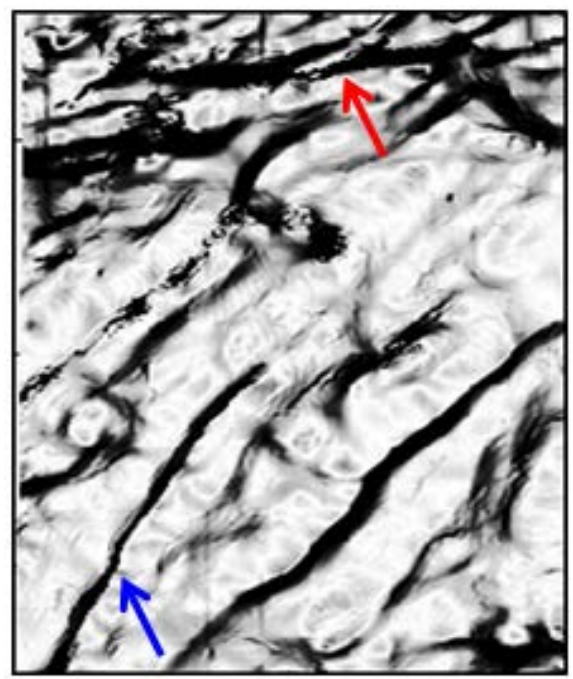

(e)

Figure 4. Single-azimuth coherence slices and an integrated coherence slice of different azimuths. (a) Azimuth 1; (b) Azimuth 2; (c) Azimuth 3; (d) Azimuth 4; (e) 4-azimuth fusion.

with new fault-block traps, were identified near those major faults. In accordance with fault properties and other conditions, some drilling sites were deployed with good results; this supported reserves and production increase in this area.

\section{Conclusions}

1) Compared with narrow-azimuth seismic data, wide-azimuth data contain more subsurface information, more illumination, and more azimuths.

2) The azimuth stacks in the directions perpendicular to fractures, which have the smallest effect of anisotropy, may improve fault interpretation.

3) Multi-azimuth data may improve fault identification and interpretation.

4) Wide-azimuth data interpretation has less uncertainties and non-uniqueness. The successful application in the Bohai Bay demonstrated its feasibility.

\section{Conflicts of Interest}

The authors declare no conflicts of interest regarding the publication of this paper.

\section{References}

Baldock, S., Tang, C. R., Beck, B., Gao, W., Cai, J., \& Hightower, S. (2012). Orthogonal Wide Azimuth Surveys: Acquisition and Imaging. First Break, 30, 35-41. https://doi.org/10.3997/1365-2397.2012012

Cordsen, A., \& Galbraith, M. (2002). Narrow-Versus Wide-Azimuth Land 3D Surveys. The Leading Edge, 21, 764-770. https://doi.org/10.1190/1.1503181

Hu, Z. P., Guan, L. P., Gu, L. X. et al. (2004) Wide Angle Seismic Wave Field Analysis and Imaging Method below the High Velocity Shield Layers. Chinese Journal of Geophys- 
ics, 47, 88-94. https://doi.org/10.1002/cjg2.459

James, K., \& Page, C. (2006). Key Aspects of Muti-Azimuth Acquisition and Processing. SEG Technical Program Expanded Abstracts, 25, 2886-2890.

Lai, W. Z. (2001). Shallow Hydrocarbon Exploration and Its Major Targets in Bohai Bay Basin. 15, 44-49.

Ling, Y. (2003). Application and Study on Wide-Azimuth Seismic Exploration. Oil Geophysical Prospecting, 38, 350-357.

Manning, T. (2007). Quantifying and Increasing the Value of Multi-Azimuth Seismic. The Leading Edge, 26, 510-520. https://doi.org/10.1190/1.2723215

Schoenberg, M. A., \& Sayers, D. S. (1999). Azimuth-Dependent Tuning of Seismic Waves Reflected from Fractured Reservoirs. Geoplhysics, 64, 1160-1171. https://doi.org/10.1190/1.1444623

Wang, X. J., Yu, B. L., Zhao, X. H., Cai, X. L., Li, H., Fang, Y., \& Liu, Y. L. (2015). Development and Application of "2W1H" Technique in Oil and Gas Exploration. China Petroleum Exploration, 20, 41-53.

Zhu, G., Wang, D. X., Liu, G. S., Song, C. Z., Xu, J. W., \& Niu, M. L. (2001) Extensional Activities along the Tan-lu Fault Zone and Its Geodynamic Setting. Chinese Journal of Ueology, 36, 269-278. 